\title{
Enhanced Cosmic Ray Flux and Ionization for Star Formation in Molecular Clouds Interacting with Supernova Remnants
}

\author{
Marco Fatuzzo, ${ }^{1}$ Fred C. Adams, ${ }^{2,3}$ and Fulvio Melia ${ }^{4}$ \\ ${ }^{1}$ Physics Department, Xavier University, Cincinnati, OH 45207 \\ ${ }^{2}$ Michigan Center for Theoretical Physics, University of Michigan \\ Physics Department, Ann Arbor, MI 48109 \\ ${ }^{3}$ Astronomy Department, University of Michigan, Ann Arbor, MI 48109 \\ ${ }^{4}$ Physics Department and Steward Observatory, The University of Arizona, AZ 85721 \\ fatuzzo@xavier.edu, fca@umich.edu, melia@physics.arizona.edu
}

\begin{abstract}
Molecular clouds interacting with supernova remnants may be subject to a greatly enhanced irradiation by cosmic rays produced at the shocked interface between the ejecta and the molecular gas. Over the past decade, broad-band observations have provided important clues about these relativistic particles and indicate that they may dominate over the locally observed cosmic-ray population by a significant amount. In this paper, we estimate the enhancement and find that the cosmic ray energy density can be up to 1000 times larger in the molecular cloud than in the field. This enhancement can last for a few Myr and leads to a corresponding increase in the ionization fraction, which has important consequences for star formation. Ionization fractions in molecular cloud cores determine, in part, the rate of ambipolar diffusion, an important process in core formation and pre-collapse evolution. Ionization fractions in newly formed circumstellar disks affect the magneto-rotational instability mechanism, which in turn affects the rate of disk accretion. As estimated here, the increased ionization acts to increase the ambipolar diffusion time by a factor of $\sim 30$ and thereby suppresses star formation. In contrast, the increased ionization fraction reduces the sizes of dead zones in accretion disks (by up to an order of magnitude) and thus increases disk accretion rates (by a comparable factor).
\end{abstract}

Subject headings: Cosmic rays - ISM: Clouds - Magnetohydrodynamics Stars: Formation - Supernovae - Turbulence 


\section{INTRODUCTION}

Although a robust paradigm for star formation within giant molecular cloud complexes now exists (Shu et al. 1987), the interplay between stars and the environment from which they are born remains poorly understood. This letter considers the interaction between supernova remnants (SNRs) and molecular clouds (MCs), and studies its effect on the level of cosmic ray fluxes within star forming regions. The cosmic ray flux affects the ionization rate, which influences the formation of molecular cloud cores and the evolution of circumstellar disks. Specifically, we explore how the cosmic ray production at the SNR/MC boundary influences the ionization fraction of the molecular gas. This ionization fraction controls the ambipolar diffusion rate (and hence the rate of core formation) and the action of magnetorotational instabilities (MRI) in circumstellar disks (and hence the rate of disk accretion).

A growing amount of data concerning the interactions of SNRs and MCs has been assembled. Huang \& Thaddeus (1986) found that about half of SNRs in the outer galaxy are spatially coincident with large molecular cloud complexes. More recently, $1720 \mathrm{MHz}$ OH maser emission, a signpost for SNR-MC interactions (Wardle \& Yusef-Zadeh 2002), has been observed from nineteen galactic SNRs - roughly $10 \%$ of the sample (Yusef-Zadeh et al. 2003). The appearance of this maser emission is important in that it helps constrain the densities and temperatures of post-shock gas in these environments. In addition, magnetic field strengths and orientations can be deduced from Zeeman splitting and polarization studies (Claussen et al. 1997; Brogan \& Troland 2001). High resolution X-ray and CO data have added to the list of interacting SNR/MC sources (Butt et al. 2001; Dubner et al. 2004; Byun et al. 2006; Sasaki et al. 2006).

Shocks produced by the interaction of SNRs and MCs can transfer $\sim 10 \%$ of the SN explosion energy into cosmic-rays (Dorfi 1999, 2000), and the interaction of these relativistic particles with the ambient medium can lead to the production of pions and, subsequently, neutral pion-decay gamma-rays (Aharonian et al. 1994). Evidence for this hadronic process has been found through the association of at least ten EGRET sources with SNRs expanding into MCs (Esposito et al. 1996; Combi et al. 1999, 2001; Torres et al. 2003); five of these sources also belong to the aforementioned set of maser SNRs. While the EGRET SNRs have gamma-ray luminosities in the $30 \mathrm{MeV}-10 \mathrm{GeV}$ band ranging from $L_{\gamma} \sim 10^{34}$ to $4 \times 10^{36}$ $\operatorname{ergs~s}{ }^{-1}$, they have not been detected at TeV energies (Rowell et al. 2000; Buckley et al. 1997). This finding sets important constraints on the distribution of energetic particles in these systems and corresponding constraints on particle-acceleration mechanisms in these high-density environments (Baring et al. 1999). These SNRs are also typically radio loud $(F \gtrsim 10 \mathrm{Jy}$ at $1 \mathrm{GHz}$ ), perhaps owing in part to synchrotron emission from secondary leptons produced via the decay of charged pions (Fatuzzo \& Melia 2003). 
Particle acceleration in the SNR/MC environments can significantly enhance the cosmicray (CR) density above that of the local background "sea" surrounding these regions (Aharonian \& Atoyan 1996, hereafter AA96; Torres et al. 2003). These energetic particles can increase the ionization fractions within the molecular cloud, and thereby influence star formation. One important process in the formation of molecular cloud cores (stellar birth sites) is the removal of magnetic flux through ambipolar diffusion. The diffusion coefficient, and the rate, depends on the ionization fraction of the cloud material. As another example, an important mechanism for producing accretion in circumstellar disks is the magneto-rotational instability (MRI) which depends on the ionization fraction in the disk. The enhanced cosmic ray flux considered here produces a corresponding increase in the ionization fraction, which in turn affects the rates of these star formation processes.

\section{COSMIC-RAY ENHANCEMENT IN SNR/MC ENVIRONMENTS}

This section estimates the enhancement of cosmic rays in SNR/MC environments (see also AA96). The EGRET SNRs have inferred gamma-ray luminosities spanning the range $L_{\gamma} \sim 10^{34}$ to $4 \times 10^{36} \mathrm{ergs} \mathrm{s}^{-1}$. This large range in luminosities may be due in part to the age distributions of the EGRET SNRs. If produced via a pion-decay mechanism, the gamma-ray luminosity initially increases as relativistic particles are continually injected into the SNR/MC environment and their population builds up. The luminosity reaches a peak once the acceleration mechanism is quenched (Sturner et al. 1996). Indeed, if $10 \%$ of the SN explosion energy is transferred to relativistic particles (Dorfi 1991, 2000), the gamma-ray luminosity of SNR/MC environments would peak at the value

$$
L_{\gamma} \sim \eta\left(\sigma_{p p} n_{p} c\right) E_{C R} \approx 2.4 \times 10^{36} \mathrm{ergs} \mathrm{s}^{-1}\left(\frac{n_{H_{2}}}{100 \mathrm{~cm}^{-3}}\right)\left(\frac{\eta}{0.1}\right)\left(\frac{E_{C R}}{10^{50} \mathrm{ergs}}\right),
$$

where $\sigma_{p p}=40 \mathrm{mb}$ is the $p p$ scattering cross-section, $\eta$ is the fraction of a relativistic proton's energy that goes into the $\pi_{0}$ decay photon channel, $E_{C R}$ is the energy content of the relativistic particles, and $n_{p}$ and $n_{H_{2}}$ are, respectively, the proton and molecular hydrogen number densities (i.e., $n_{p}=2 n_{H_{2}}$ ). ${ }^{1}$ The value of $\eta$ can be estimated using the approximation for the gamma-ray luminosity due to the decay of neutral pions

$$
L_{\gamma} \approx \frac{2 \Lambda^{0}(\alpha)}{\alpha}\left(\sigma_{p p} n_{p} c\right) \int E_{\gamma} \frac{d N_{C R}\left(E_{\gamma}\right)}{d E} d E_{\gamma} \approx \frac{2 \Lambda^{0}(\alpha)}{\alpha}\left(\sigma_{p p} n_{p} c\right) E_{C R},
$$

\footnotetext{
${ }^{1}$ Molecular clouds are highly nonuniform, with densities on different scales ranging over several orders of magnitude. However, the mean molecular hydrogen density of MCs is observed to be $\sim 10^{2} \mathrm{~cm}^{-2}$
} 
where $\alpha$ is the spectral index of the distribution $d N_{C R} / d E$ of relativistic protons, and $\Lambda^{0}$ is a dimensionless parameter that depends on $\alpha$ (Crocker et al. 2005). The above equations imply $\eta=2 \Lambda^{0} / \alpha$, so that $\eta=0.18,0.10$, and 0.60 for $\alpha=2.0,2.2$, and 2.4 , respectively. The populations of relativistic protons injected into SNR/MC environments are expected to have power-law distributions with $\alpha=2-2.4$ (Jones \& Ellison 1991), in good agreement with the EGRET observations. Since the diffusion rate depends on the energy of the particles, the spectrum of relativistic protons may flatten inside a MC so that $\Delta \alpha \approx 0.2$ (Ballantyne et al. 2006). Since the ionization rate depends on the number density of particles (rather than the energy density), this change in spectral index implies a corresponding change in number density (by a factor of $\sim 2$ ), but the exact size of the change depends on the details of the diffusion process. In future work, a careful assessment of this effect should be carried out.

Next we assume that particles build up during an injection phase to their peak values on a timescale of $\sim 10^{4}$ years, consistent with estimates of the EGRET SNR ages. A fraction of these particles will then diffuse into the cloud with which the SNR is interacting (where the fraction is determined by the solid angle subtended by the cloud). Diffusion continues until the cosmic rays undergo $p p$ scattering with the ambient medium, and thereby experience catastrophic losses, on a time scale given by

$$
\tau_{p p} \equiv\left(\kappa \sigma_{p p} n_{p} c\right)^{-1} \approx 3 \times 10^{5} \mathrm{yrs}\left(\frac{n_{H_{2}}}{10^{2} \mathrm{~cm}^{-3}}\right)^{-1}
$$

where $\kappa \approx 0.45$ accounts for the inelasticity of $p p$ interactions. Since protons only lose a fraction of their energy in a given scattering event, the total time of the cosmic ray enhancement could be larger than $\tau_{p p}$ by a factor of 2 or 3 . Setting the time scale $\tau_{p p}$ equal to the diffusion time scale $\tau_{d i f} \equiv R^{2} / D(E)$, where $D(E)$ is the energy-dependent diffusion coefficient, one can estimate the distance $R(E)$ that particles will diffuse into the cloud before scattering and losing their energy. Adopting the expression $D(E)=D_{10}(E / 10 \mathrm{GeV})^{1 / 2}$, where $D_{10}=10^{28}$ $\mathrm{cm}^{2} \mathrm{~s}^{-1}$ (AA96), we find

$$
R(E)=\sqrt{\tau_{p p} D(E)} \approx 100 \mathrm{pc}\left(\frac{n_{H_{2}}}{10^{2} \mathrm{~cm}^{-3}}\right)^{-1 / 2}\left(\frac{D_{10}}{10^{28} \mathrm{~cm}^{2} \mathrm{~s}^{-1}}\right)^{1 / 2}\left(\frac{E}{10 \mathrm{GeV}}\right)^{1 / 2} .
$$

As noted in AA96, however, values of $D_{10} \sim 10^{26} \mathrm{~cm}^{2} \mathrm{~s}^{-1}$ may be more applicable in the molecular cloud environment (Ormes et al. 1988), leading to an estimate of $R(10 \mathrm{GeV})=$ 10 pc. This length scale is comparable to the typical sizes of molecular clouds.

The energy loss rate for a cosmic-ray due to ionization is weakly dependent on energy for relativistic protons, and is given by $d E / d t \sim 3.6 \times 10^{-7} n_{H_{2}} \mathrm{eV} / \mathrm{s}$ (Mannheim \& Schlickeiser 1994). The corresponding cooling time thus takes the form

$$
\tau_{c i} \approx 8.8 \times 10^{6} \mathrm{yrs}\left(\frac{E}{10 \mathrm{GeV}}\right)\left(\frac{n_{H_{2}}}{10^{2} \mathrm{~cm}^{-3}}\right)^{-1}
$$


which is considerably longer than the $p p$ collision time for all but the least energetic protons. The resulting energy density $u_{c r}$ within the MC during the SNR/MC interaction will be $u_{c r} \sim 500 \mathrm{eV} \mathrm{cm}^{-3}\left(E_{C R} / 10^{50} \mathrm{ergs}\right)(R / 10 \mathrm{pc})^{-3}$. This value is about $10^{3}$ times larger than the energy density of the local cosmic-rays (where $u_{c r} \sim 0.5 \mathrm{eV} \mathrm{cm}^{-3}$ ). Thus, a significant enhancement of cosmic ray energy density can be achieved in molecular clouds through their interactions with SNRs.

\section{ENHANCEMENT OF THE IONIZATION FRACTION}

The number density of ionized particles in a $\mathrm{MC}$ environment depends on the ionization rate (due to cosmic-rays) and a complex recombination process. For steady-state conditions, the ionization fraction $x \equiv n_{e} / n_{H_{2}}$ is well approximated (Elmegreen 1979; Shu 1983, 1992) by the expression

$$
x \sim 10^{-5}\left(\zeta / 10^{-17} \mathrm{~s}^{-1}\right)^{1 / 2}\left(n / 10^{2} \mathrm{~cm}^{-3}\right)^{-1 / 2} .
$$

This expression is valid for $n \lesssim 10^{6} \mathrm{~cm}^{-3}\left(\zeta / 10^{-17} \mathrm{~s}^{-1}\right)$. Assuming that $\zeta$ scales linearly with the cosmic ray energy density, i.e., $\zeta=10^{-17} \mathrm{~s}^{-1}\left(u_{c r} / 0.5 \mathrm{eV} \mathrm{cm}^{-3}\right)$, the ionization fraction in molecular clouds interacting with SNRs can be enhanced by up to a factor of $\sim 30$. This scaling is strictly valid when $n \lesssim 10^{9} \mathrm{~cm}^{-3}$ for the enhanced cosmic ray flux considered here. The process of ambipolar diffusion occurs primarily at lower densities, and will be fully affected by this enhancement. In circumstellar disks, however, the densities are larger near the disk midplane, and the full ionization enhancement might not be realized. Notice that the ionization rate depends on the number density of cosmic rays, rather than the energy density. If the spectrum $d N_{C R} / d E$ is invariant, the two quantities scale together. However, the index $\alpha$ of the spectrum could vary $(\S 2)$, so that the scaling could differ by a factor of $\sim 4$ (which affects the ionization fraction by a factor of 2 ).

The timescale $\tau_{i}$ required to reach this enhancement level can be estimated by setting the ionization rate $\dot{n}_{e}=\zeta n$ equal to the ratio $n_{e} / \tau_{i}$. Using the expression for the ionization fraction $x$ given by equation (6), this timescale estimate becomes $\tau_{i}=x / \zeta \approx 3 \times 10^{4} \mathrm{yr}$ $\left(\zeta / 10^{-17} \mathrm{~s}^{-1}\right)^{-1 / 2}\left(n / 10^{2} \mathrm{~cm}^{-3}\right)^{-1 / 2}$, only about $1000 \mathrm{yr}$ when $\zeta \sim 10^{-14} \mathrm{~s}^{-1}$. The ionization enhancement is thus established at virtually the same time as the CR enhancement. Of course, this result also means that the ionization enhancement will decay on a comparably short timescale, as soon as the CR enhancement is removed due to $p p$ scattering (up to 1 Myr, as estimated in $§ 2$; this time can be extended, as discussed below).

The discussion so far considers the MC environment to be uniform. However, these MC regions are highly nonuniform, exhibiting hierarchical structure that can be characterized in terms of dense clumps and cores surrounded by an interclump gas with $n \sim 5-25 \mathrm{~cm}^{-3}$. 
Clumps have characteristic densities $n \sim 10^{3} \mathrm{~cm}^{-3}$ and radii $r \approx 0.1-1$ pc. These dense regions occupy a relatively small fraction $(2-8 \%)$ of the cloud volume, but can account for most of its mass (Williams et al. 1995). As a result, cosmic rays propagating through the $\mathrm{MC}$ environment spend most of their time in a lower density medium $\left(n_{\mathrm{H}_{2}} \sim 10 \mathrm{~cm}^{-3}\right)$, so the $p p$ collision timescale would be $\sim 3$ Myr. Of course, this point is somewhat diminished by the fact that these particles also spend a small fraction of their life $(\sim 2-8 \%)$ in the denser regions. If these particles can survive without undergoing $p p$ scattering for $\sim 1 \mathrm{Myr}$, the enhancement in cosmic ray energy density can last for a few Myr.

The interaction of relativistic protons with the MC medium leads to the production of both neutral and charged pions, with each species produced in equal numbers. As a result, $p p$ scattering transfers roughly $3 \%$ of the initial cosmic-ray energy to secondary electrons and positrons - the byproducts of charged pion decays. The injection of these leptons can increase the energy density of cosmic-rays above the field by as much as a factor of $\sim 30$, and increases the ionization fraction by a factor of $\sim 5$ (assuming that $\zeta \propto u_{c r}$ ). The peak of the injected secondary lepton distribution occurs at $\sim 60 \mathrm{MeV}$. Near this peak energy, leptons traversing neutral atomic hydrogen cool predominantly via electronic excitation on a timescale $\tau_{c} \sim 7 \times 10^{4}\left(n_{p} / 10^{2} \mathrm{~cm}^{-3}\right)^{-1}$ yr (Gould 1975). The enhancement in the ionization fraction due to secondary leptons would therefore only last for a comparably short time.

\section{EFFECTS ON STAR FORMATION}

The enhancement in cosmic ray flux, which increases ionization levels, can have a significant impact on star formation. Ionization affects the coupling between magnetic fields and the (largely neutral) gas. On scales of $\sim 0.1 \mathrm{pc}$, increased ionization leads to greater coupling between gas and the magnetic fields, and acts to slow down star formation. On smaller scales of $\sim 1 \mathrm{AU}$, increased ionization in circumstellar disks allows for MRI to remain active over a greater extent of the disk and thereby allows for larger disk accretion rates.

In considerations of ambipolar diffusion, the density of ions takes the form $\rho_{i}=\mathcal{C} \rho_{n}^{1 / 2}$ because the volumetric rate of recombinations is proportional to $n_{e} n_{i} \sim n_{i}^{2}$ and the volumetric rate of ionization is proportional to $\zeta n_{n}$ (Shu 1992). As a result, the constant $\mathcal{C} \propto \sqrt{\zeta}$. Standard considerations of ambipolar diffusion (Mouschovias 1976; Shu 1983, 1992) show that the effective diffusion constant is given by $D \sim v_{A}^{2} /(\gamma \mathcal{C} \rho) \propto \zeta^{-1 / 2}$, where $v_{A}$ is the Alfvén speed and $\gamma$ is the drag coefficient between ions and neutrals. As the cosmic ray flux increases, the diffusion coefficient decreases as the square root of the flux. A 1000-fold increase in the cosmic ray flux (\$2) thus decreases the effective diffusion constant by a factor of $\sim 30$, and increases the ambipolar diffusion time scale by the same factor. Current observations 
indicate that the diffusion process is too slow to account for the observed statistics of starless molecular cloud cores (e.g., Jijina et al. 1999), so that some mechanism to speed up the process is required, even in the absence of supernova enhancement of the cosmic ray flux (Ciolek \& Basu 2001, Zweibel 2002, Fatuzzo \& Adams 2002). The factor of $\sim 30$ increase in timescale due to cosmic ray enhancement essentially shuts down the process. Although supernovae have often been invoked as a means to trigger star formation (Elmegreen 1998), this mechanism provides a channel for supernovae to inhibit star formation.

An enhancement in cosmic ray flux also influences disk accretion for star/disk systems associated with the interaction region. A growing consensus in the field holds that disk accretion is produced by an effective viscosity that is driven by turbulence, which in turn is driven by MHD instabilities such as MRI (Balbus \& Hawley 1991). In order for MRI to operate, and hence for disk accretion to take place, the ionization fraction must be sufficiently high so that the gas is well coupled to the field. The inner disk can be ionized by collisions (where the number density and temperature are high), and the outer disk can be ionized by standard values of the cosmic ray flux, but intermediate regions may have dead zones where ionization is too low (Gammie 1996). The enhanced cosmic ray flux acts to increase the fraction of the disk that is active, i.e., sufficiently ionized for MRI to operate.

To illustrate the possible effects of an enhanced cosmic ray flux, consider the disk models of Gammie (1996), where the cosmic ray flux has its standard (unenhanced) value. The flux is assumed to be ineffective as an ionization source after it falls to 1 /e times its initial value. The attenuation column density for cosmic ray ionization is $\Sigma_{0} \approx 100 \mathrm{~g} / \mathrm{cm}^{2}$ (Umebayashi \& Nakano 1981). As a result, only the uppermost $100 \mathrm{~g} / \mathrm{cm}^{2}$ of the disk (and a corresponding layer on the other side) experience enough cosmic ray ionization for MRI to operate. With a cosmic ray flux that is enhanced by a factor $\mathcal{F}$, the disk will experience cosmic ray ionization down to a larger column density $\Sigma_{*} \approx(1+\ln \mathcal{F}) \Sigma_{0}$. For an optimal enhancement of $\mathcal{F} \approx 1000$, the active column density becomes $\Sigma_{*} \approx 8 \Sigma_{0} \approx 800 \mathrm{~g} / \mathrm{cm}^{2}(\sim 8$ times more disk material can be ionized). To put this result in perspective, consider a circumstellar disk with mass $M_{d}=0.05 M_{\odot}$, radius $r_{d}=30 \mathrm{AU}$, and surface density profile $\Sigma \propto 1 / r$. The total surface density at $1 \mathrm{AU}$ is thus $\Sigma(1 \mathrm{AU}) \approx 2400 \mathrm{~g} / \mathrm{cm}^{2}$. Since the upper and lower $800 \mathrm{~g} / \mathrm{cm}^{2}$ of the column can be ionized with an enhanced cosmic ray flux, about $2 / 3$ of the disk can remain active at $r=1 \mathrm{AU}$. As a result, the dead zone subtends only the central $33 \%$ of the disk column density, compared with $92 \%$ for no cosmic ray enhancement. Since both the disk properties and the cosmic ray flux enhancement will vary from system to system, an extensive exploration of parameter space is warranted. Nonetheless, this calculation illustrates that supernova enhancements of the cosmic ray flux can have a substantial impact on disk accretion. In the most extreme case, the disk accretion rate can be an order of magnitude larger than in regions with no cosmic ray enhancement. 


\section{CONCLUSIONS}

This letter has shown that a supernova interacting with a molecular cloud can produce a substantial enhancement in the flux of cosmic rays within the cloud. These cosmic rays can diffuse a distance $R \sim 10$ pc into the molecular cloud before undergoing $p p$ scattering leading to catastrophic energy losses. The resulting flux enhancement is estimated to be a factor of $\sim 10^{3}(R / 10 \mathrm{pc})^{-3}$ within the diffusion region, and can last for a few Myr after cosmic-ray acceleration ceases. The corresponding enhancement in the ionization fraction within the molecular cloud is estimated to be $\sim 30(R / 10 \mathrm{pc})^{-3 / 2}$. This cosmic-ray enhancement may have a significant impact on star formation processes, primarily by affecting the ionization fraction. The enhanced ionization fraction in MC cores, the sites of protostellar collapse, increases the ambipolar diffusion time scale by a factor of $\sim 30$, so that supernovae inhibit further star formation via this mechanism. The enhanced cosmic ray flux also increases the ionization levels within circumstellar disks. In this setting, more of the disk can actively support MRI, so that supernovae enhancement of cosmic ray flux leads to increased rates of disk accretion, by a factor of $\sim 8$.

During the past decade, multiwavelength observations have provided important clues about cosmic ray acceleration resulting from the interaction between supernova remnants and molecular clouds. While $\mathrm{OH}$ maser emission has helped constrain the environment of the acceleration sites, EGRET observations have helped constrain the resulting particle distributions. Additionally, since the gamma-ray emission above $\sim 100 \mathrm{MeV}$ is believed to result primarily from the decay of neutral pions produced by the scattering of the cosmic rays with the ambient medium, future GLAST observations promise to advance our understanding of how cosmic rays diffuse from their acceleration sites and interact with the molecular cloud environment. Along with these future observations, additional theoretical work should be carried out to explore the details of the diffusion of cosmic rays into molecular clouds, the enhanced ionization fractions produced therein, and the subsequent effects on star formation.

This work was supported by Xavier University through the Hauck Foundation. This work was supported at the University of Michigan by the Michigan Center for Theoretical Physics, and by NASA through the Astrophysics Theory Program (NNG04GK56G0) and the Spitzer Space Telescope Theoretical Research Program. This work was supported at The University of Arizona by NSF (AST-0402502). 


\section{REFERENCES}

Aharonian, F. A., Drury, L. O'C, and Völk, H. J. 1994, A\&A, 285, 645

Aharonian, F. A. \& Atoyan, A. M. 1996, A\&A, 309, 917 (AA96)

Aharonian, F. A., 2001, SSRv, 99, 187

Balbus, S., \& Hawley, J. 1991, ApJ, 376, 214

Ballantyne, R. D., Melia, F., \& Liu, S. 2006, submitted to ApJ Letters

Baring, M. G., Ellison, D. C., Reynolds, S. P., Greiner, I. A., \& Goret, P. 1999, ApJ, 513, 311

Brogan, C. L., \& Troland, T. H. 2001, ApJ, 550, 799

Buckley, J. H., et al. 1998, A\&A, 329, 639

Butt, Y. M., Torres, D. F., Combi, J. A., Dame, T., \& Romero, G. E. 2001, ApJ, 562, L167

Byun, D.-Y., Koo, B.-C., Tatematsu, K., \& Sunada, K. 2006, ApJ, 637, 283

Ciolek, G. E., \& Basu, S. 2001, ApJ, 547, 272

Claussen, M. J., Frail, D. A., Goss, W. M., \& Gaume, R. A. 1997, ApJ, 489, 143

Combi, J. A., Romero, G. E., Benaglia, P. 1998, A\&A, 333, L91

Combi, J. A., Romero, G. E., Benaglia, P., \& Jonas, J. L. 2001, A\&A, 366, 1047

Crocker, R. M., Fatuzzo, M., Jokipii, J. R., Melia, F., \& Volkas, R. R. 2005, ApJ, 622, 892

Dorfi, E. A., 1991, A\&A, 251, 597

Dorfi, E. A., 2000, ApSS, 272, 227

Dubner, G., Giacani, E., Reynoso, E., \& Parõn, S. 2004, A\&A, 426, 201

Elmegreen, B. G. 1974, ApJ, 232, 729

Elmegreen, B. G. 1998, ASP Conf. Ser. 148: Origins, eds. C. E. Woodward, J. M. Shull, and H. A. Thronson, p. 150

Esposito, J. A., Hunter, S. D., Kanback, G., \& Streekumar, P. 1996, ApJ, 461, 820

Fatuzzo, M., \& Adams, F. C. 2002, ApJ, 570, 210 
Fatuzzo, M., \& Melia, F. 2005, ApJ, 630, 321

Gammie, C. F. 1996, ApJ, 457, 355

Gould, R. J. 1975, ApJ, 196, 689

Huang, Y.-L., \& Thaddeus, P. 1986, ApJ, 309, 804

Jijina, J., Myers, P. C., \& Adams, F. C. 1999, ApJS, 125, 161

Jones, F., \& Ellison, D. 1991, Space Sci. Rev., 58, 259

Mannheim, K. \& Schlickheiser, R. 1994, A\&A, 286, 983

Mouschovias, T. Ch. 1976, ApJ, 207, 141

Ormes, J. F., Ösel, M. E., Morris, D. J. 1988, ApJ, 334, 722

Romero, G. E., Benaglia, P., \& Torres, D. F. 1999, A\&A, 348, 868

Rowell, G. P., et al. 2000, A\&A, 359, 337

Sasaki, M., Kothes, R., Plucinsky, P. P., Gaetz, T. J., \& Brunt, C. M. 2006, ApJ, 642, L149

Shu, F. H. 1983, ApJ, 273, 202

Shu, F. H. 1992, Gas Dynamics, (Mill Valley: Univ. Sci. Books)

Shu, F. H., Adams, F. C., \& Lizano, S. 1987, ARA\&A, 25, 23

Sturner, S. J., Skibo, J. G., Dermer, C. D., \& Mattox, J. R. 1997, ApJ, 490, 619

Torres, D. F., et al. 2003, PhR, 382, 303

Umeybayashi, T., \& Nakano, T. 1981, PASJ, 33, 617

Wardle, M., \& Yusef-Zadeh, F. 2002, Science, 296, 2350

Williams, J. P., Blitz, L., \& Stark, A. A. 1995, ApJ, 451, 252

Yusef-Zadeh, F., Wardle, M., Rho, J., \& Sakano, M. 2003, ApJ, 585, 319

Zweibel, E. G. 2002, ApJ, 567, 962 